\title{
Hydrodynamique sédimentaire sur le plateau continental du golfe du Lion
}

\author{
Yann LEREDDE ${ }^{1}$, Héloïse MICHAUD ${ }^{1}$ \\ ${ }^{1}$ Géosciences Montpellier, Géosciences Montpellier, UMR 5243 - CC 60, \\ Université Montpellier 2, Place E. Bataillon, 34095 Montpellier cedex 5, France. \\ leredde@gm.univ-montp2.fr
}

\section{Résumé :}

L’hydrodynamique du plateau continental du Golfe du Lion est généralement induite par les vents. Les simulations numériques réalisées par les modèles numériques de circulation océanique côtière aux équations primitives sont en général cohérentes avec les observations in-situ. Très peu d’observations étaient toutefois disponibles pendant les épisodes de tempêtes méditerranéennes.

Aussi, la station BESSète (Bottom Experimental Station Sète), équipée d'un ADCP mesurant en continu les caractéristiques de houle et les profils verticaux de courants, a été implantée sur le plateau sous $70 \mathrm{~m}$. Les premières données issues de cette station, acquises en février-mars 2007, confirment les schémas de circulations induits par les vents de Nord (Mistral, Tramontane). Un épisode de tempête $(\mathrm{Hs}>5 \mathrm{~m})$ a aussi pu être observé le 18 février 2007. De forts courants dont l'intensité peut atteindre $0,7 \mathrm{~ms}^{-1} \mathrm{y}$ sont associés.

Cette série de données est ensuite utilisée pour calculer les tensions de cisaillements induites sur le fond. Sous $70 \mathrm{~m}$, elles sont en général quasi-nulles excepté pendant l'épisode de tempête où l'action conjuguée des houles et des courants peut induire des contraintes susceptibles de mobiliser et déplacer le sédiment fin présent dans la zone.

La très forte intensité des courants mesurés pendant la tempête amènent ensuite à reconsidérer les processus de transferts de quantité de mouvement entre atmosphère, océan et sédiments. Très schématiquement, sur le plateau, dès que la houle interagit avec le fond, la houle se transforme (réfraction, dissipation) et transmettrait de la quantité de mouvement à l'échelle des courants moyens. Cette hypothèse est retenue pour expliquer ces forts courants observés et pourrait être confirmée par d’autres travaux théoriques et numériques.

\section{Mots-clés :}

Houle - Courant - Sédiment - Tempête - ADCP- Plateau continental 


\section{Introduction}

L'hydrodynamique du plateau continental du golfe du Lion est généralement induite par les flux atmosphériques qui agissent localement, par exemple pour former des upwellings (Millot, 1990), ou à l'échelle du plateau pour former des structures tourbillonnaires pouvant interagir avec la circulation générale méditerranéenne (PETRENKO et al., 2005). Cette hydrodynamique est très variable et parfois difficile à schématiser de façon simple. La réponse hydrodynamique aux conditions météorologiques s’avère parfois peu intuitive. Les modèles numériques de circulation océanique côtière aux équations primitives, tels que les modèles Symphonie (ESTOURNEL et al., 2003) ou Mars3D (ANDRE et al., 2005), placés dans des conditions réalistes (notamment les forçages atmosphériques et de grande échelle donnés par des systèmes préopérationnels), sont toutefois bien représentatifs de la grande majorité des circulations océaniques observées. Par exemple, les travaux de LEREDDE et al. (2007) menés sur la zone nord du plateau montrent comment le modèle numérique Symphonie reproduit bien les observations réalisées principalement par ADCP de coque sur 9 jours de campagne et comment en retour la vision complète apportée par le modèle permet de mieux comprendre ces observations. La zone géographique étudiée, le golfe d’Aigues-Mortes, est représentée sur la figure 1. Réalisées sous conditions de vents de Nord (Tramontane et Mistral), de temps calme ou de faibles vents de Sud-Est, ces observations et ces modélisations montrent des courants de surface qui ne dépassent jamais $0,4 \mathrm{~ms}^{-1}$ et qui, au fond, sont modélisées à des valeurs inférieures à $0,2 \mathrm{~ms}^{-1}$.

Cette hydrodynamique, principalement induite par les vents, engendre rarement d'importants remaniements sédimentaires, les tensions de cisaillement exercées sur le fond restant très faibles. Ces remaniements et ces cisaillements de fond sont en réalité susceptibles de devenir importants lors des épisodes de tempêtes induisant de fortes houles. Ces épisodes sont néanmoins très difficiles à observer. Ils nécessitent en effet la mise en place de stations implantées à demeure. Dans le golfe du Lion, de telles expériences ont été menées dans des zones relativement proches du littoral. Les tempêtes de décembre 1999 dans la baie de Banyuls (GREMARE et al., 2003) et de février 2004 à l'embouchure de la Têt (GUILLEN et al., 2006) ont ainsi pu être observées sous des fonds de $30 \mathrm{~m}$. DENAMIEL (2006) montre que les forts courants observés pourraient être induits par le transfert de quantité de mouvement apportée par les très fortes houles observées. Ces deux stations, relativement proches du littoral, n'étaient toutefois pas complètement représentatives de la circulation océanique induite sur le plateau lors des épisodes de tempête. Une nouvelle station de mesures a donc été 
implantée sous $70 \mathrm{~m}$ de fond, dans la zone où les houles de tempête commencent à interagir avec le fond. Cette station, nommée BESSète (Bottom Experimental Station Sète), est équipée d'un ADCP mesurant en continu les caractéristiques de houle et les profils verticaux de courants.

Dans cet article, nous présentons les premières données issues de cette station. Il s'agit d'une première série temporelle acquise en février-mars 2007. Les conditions atmosphériques sont principalement des vents de Nord (Tramontane et Mistral) mais nous avons également eu la chance de capter un épisode de tempête de Sud-Est avec une houle de hauteur significative supérieure à $5 \mathrm{~m}$. Ces données servent d'une part à estimer les contraintes de cisaillement exercées sur le fond et d'autre part à initier une discussion sur les transferts de quantité de mouvement de l'atmosphère vers les sédiments en passant par l’océan.

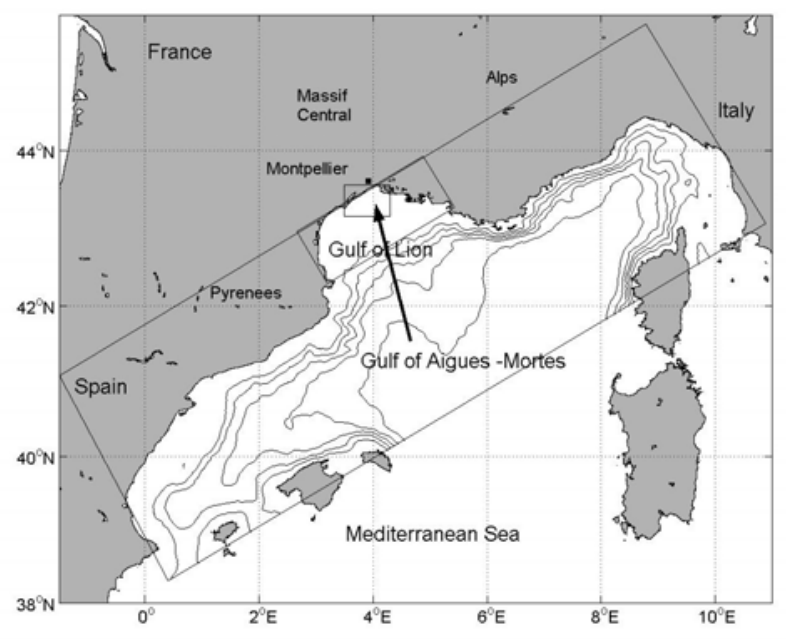

Figure 1. Localisation du site d'étude. Domaines de modélisations emboîtées. D'après LEREDDE et al, 2007.

\section{La station BESSète}

\subsection{Dispositif expérimental}

La station BESSète (Bottom Experimental Station Sète) (voir figure 2) est une station automatique de mesure de courants et de houle implantée sous $70 \mathrm{~m}$ de bathymétrie au sud-est de Sète. Située à la position $3^{\circ} 50^{\prime} \mathrm{E}, 43^{\circ} 19^{\prime} \mathrm{N}$, cette station est équipée d'un ADCP de fond (RDI $300 \mathrm{kHz}$ ) complété par un module de houle, un socle anti-chalutage et un système de largueur acoustique. Cette station posée sur le fond est protégée en surface par une bouée (bouée d’Eaux Saines) du SMNLR (Service Maritime Navigation Languedoc-Roussillon). 
Placée en pleine zone de pêche " intensive » par chalutages, et même si une grosse bouée de signalisation limite les passages, une cage de protection antichalutage de type TRBM (Trawl Resistant Bottom Mount) a spécialement été acquise auprès de Flotation Technologies (voir photo sur la figure 2). La mise à l'eau se fait en descendant le TRBM au portique. Posé au fond, il est libéré du câble de descente par un largueur acoustique. La station est autonome et sa durée d'autonomie dépend de la capacité des batteries de l'ADCP. Un pack externe de batteries a été ajouté mais la mesure à haute fréquence $(2 \mathrm{~Hz})$ nécessaire pour enregistrer les caractéristiques de houle limite l'autonomie. L'ADCP est actuellement configuré pour mesurer les courants toutes les $1 / 2$ heures avec une résolution verticale de $2 \mathrm{~m}$, et les caractéristiques de houle pour des « bursts » de $8 \mathrm{mn}$ toutes les 3 heures. Cela donne une autonomie de 7 mois.

La récupération se fait en déclenchant un largueur acoustique contenu dans le recovery pod (partie orange sur la figure 2) dont la flottabilité est positive. Une «laisse» permet de remonter le socle de mouillage. Ces opérations nécessitent un minimum de beau temps. Des données hydrodynamiques dans de telles zones sont très rares et cela constitue aussi un challenge technologique de maintenir cette station.

\subsection{Données de courants et de caractéristiques de houle}

La station BESSète a été pour la première fois mouillée le 12 février 2007 et récupérée le 25 mars 2007. La figure 3 montre cette première série temporelle de données mesurées de caractéristiques de houle et de courants. Ces données sont mises en relation avec la série temporelle de vents mesurés à la station météorologique de Sète.

Cette période de fin d'hiver est marquée par l'omniprésence de vents de Nord, principalement de Nord-Ouest (Tramontane) à Nord-Est (Mistral). Ces vents peuvent engendrer des vagues courtes de faibles amplitudes. Les courants enregistrés sont en grande majorité parallèles aux isobathes (vers le Nord-Est ou le Sud-Ouest) avec des intensités restant inférieures à $0,3 \mathrm{~ms}^{-1}$. Ces données confirment bien les observations acquises pendant les surveys par ADCP de coque des campagnes HYGAM (LEREDDE et al., 2007). LEREDDE et al. (2007) montrent également que de telles circulations sont reproduites de manière très réaliste par un modèle numérique de circulation océanique côtière comme Symphonie (ESTOURNEL et al., 2003). Ces vents de Nord ont des effets locaux bien connus (par exemple des upwellings, (MILLOT, 1990)) mais agissent également à l'échelle de l'ensemble du plateau continental pour former des structures tourbillonnaires de grande échelle ayant une permanence de quelques jours (ESTOURNEL et al., 2003). 


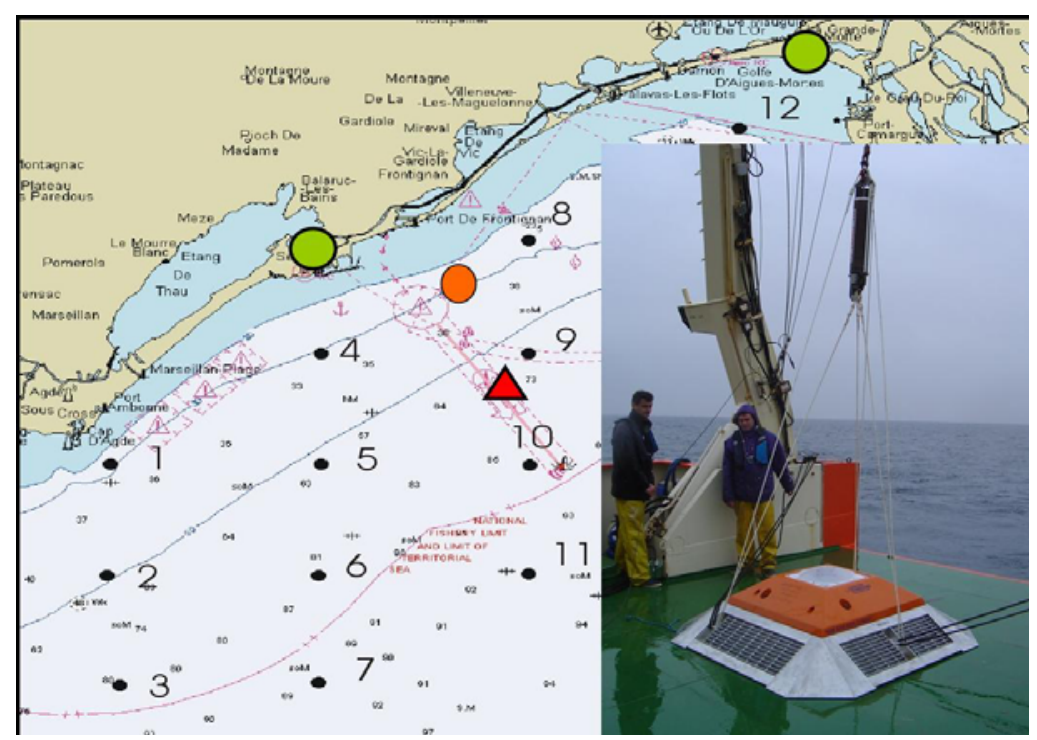

Figure 2. Position (triangle rouge) et photographie de la station BESSète et de son dispositif de mise à l'eau sur le pont du NO Tethys II avant sa première mise à l'eau le 12 février 2007. Les points numérotés correspondent à des stations CTD. Le point orange correspond à la position de la bouée houlographe du SMNLR. Les deux points verts correspondent à la position des principales stations météorologiques utilisées dans cette étude (Sète et La Grande Motte).

L’intérêt supplémentaire de cette série de données concerne l'épisode de tempête du 18 février 2007. Un tel épisode n'est observable que si l'on dispose d'instruments préinstallés in-situ. En effet, même quand le navire est programmé pendant un tel épisode, de telles mesures sont impossibles. Cela a été le cas pour la campagne HYGAM07-6 programmée du 20 au 22 novembre 2007, journées marquées par une très forte tempête de Sud où la sortie en mer a été impossible. L'épisode de tempête du 18 février 2007 a, quant à lui, pu être observé grâce à la station BESSète. Les vents de Sud-Est locaux, mesurés à Sète, sont restés modérés (10 à $15 \mathrm{~ms}^{-1}$ ) mais les houles générées sur l'ensemble de la Méditerranée Nord-Occidentale ont atteint des hauteurs significatives supérieures à $5 \mathrm{~m}$. Les courants mesurés de manière concomitante ont atteint des intensités supérieures à $0,8 \mathrm{~ms}^{-1}$ en surface et à $0,6 \mathrm{~ms}^{-1}$ sur toute la colonne d'eau. La figure 4 montre le profil vertical de courant mesuré à l'apex de la tempête. De telles intensités de courants sont très fortes. Que ce soit dans les observations par l'ADCP de coque du NO Tethys II lors des campagnes HYGAM ou que ce soit dans des simulations numériques Symphonie, les intensités de courants ne dépassent pas $0,4 \mathrm{~ms}^{-1}$.

Si on réalise une simulation numérique forcée par un vent correspondant au vent observé (Sud-Est à $15 \mathrm{~ms}^{-1}$ ), la tension de cisaillement exercée en surface est égale à $0,4 \mathrm{Nm}^{-2}$ et on obtient des intensités de courant de l'ordre de $0,2 \mathrm{~ms}^{-1}$. Par un petit exercice variationnel, si on essaie de maximiser ces courants simulés pour 
que leurs valeurs se rapprochent des courants observés, on est amené à placer le modèle Symphonie sous une contrainte de cisaillement de surface exagérément forte d'intensité égale $2.8 \mathrm{Nm}^{-2}$ et de direction Est-Nord-Est. De telles contraintes de cisaillement de surface ne sont plus physiquement réalistes et de plus, les courants simulés ne sont pas encore suffisamment forts (voir figure 4).

De telles mesures de courants amènent à réfléchir à la physique prise en compte dans les modèles numériques de circulation océanique côtière comme Symphonie et, au-delà, aux processus qui induisent de tels courants pendant les tempêtes (voir chapitre 3.)
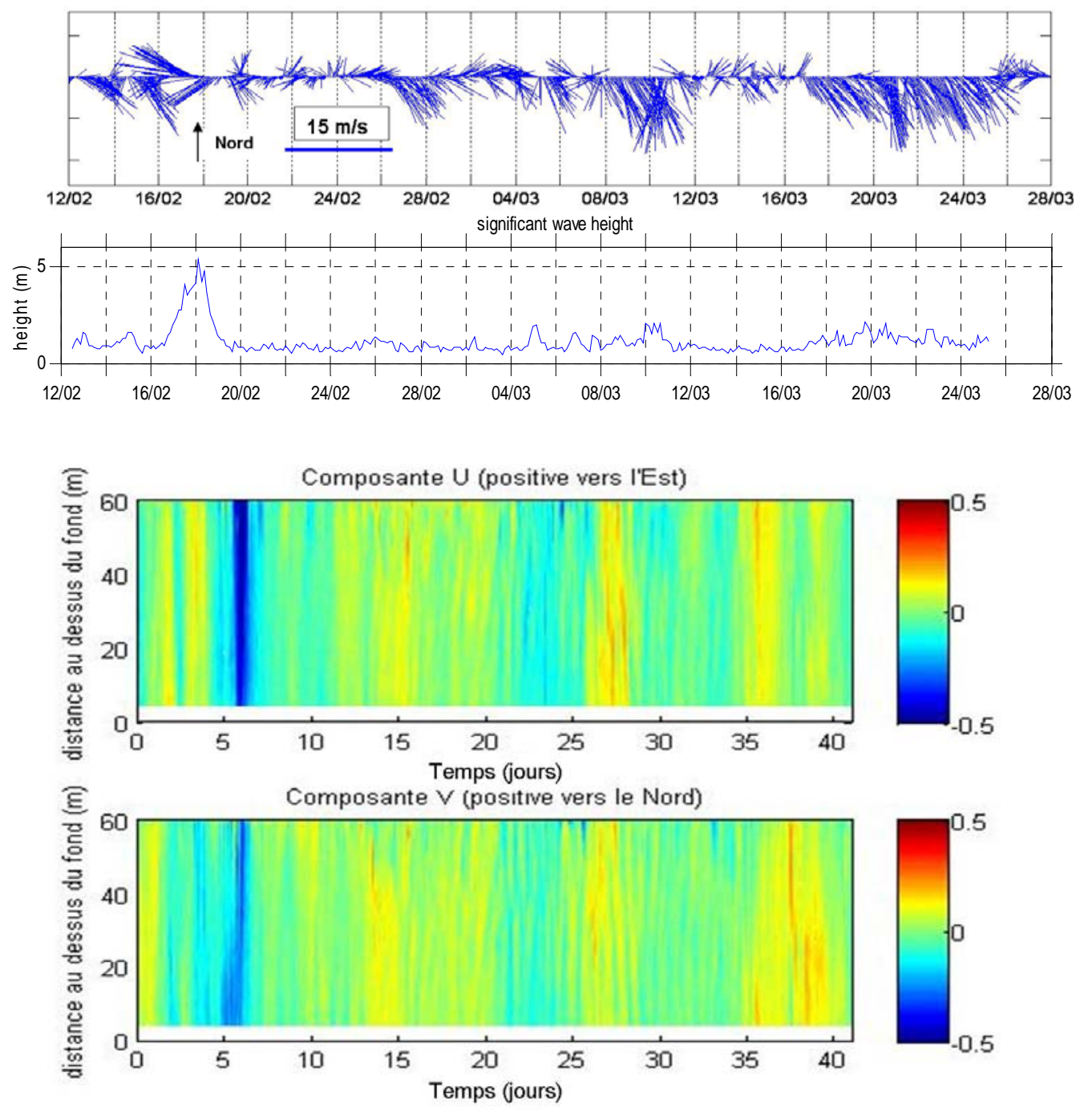

Figure 3. Série temporelle de données mesurées entre le 12 février et le 25 mars 2007. Vents à la station météorologique de Sète (haut). Hauteur significative de la houle mesurée à la station BESSète (milieu). Courants mesurés à la station BESSète (bas). 


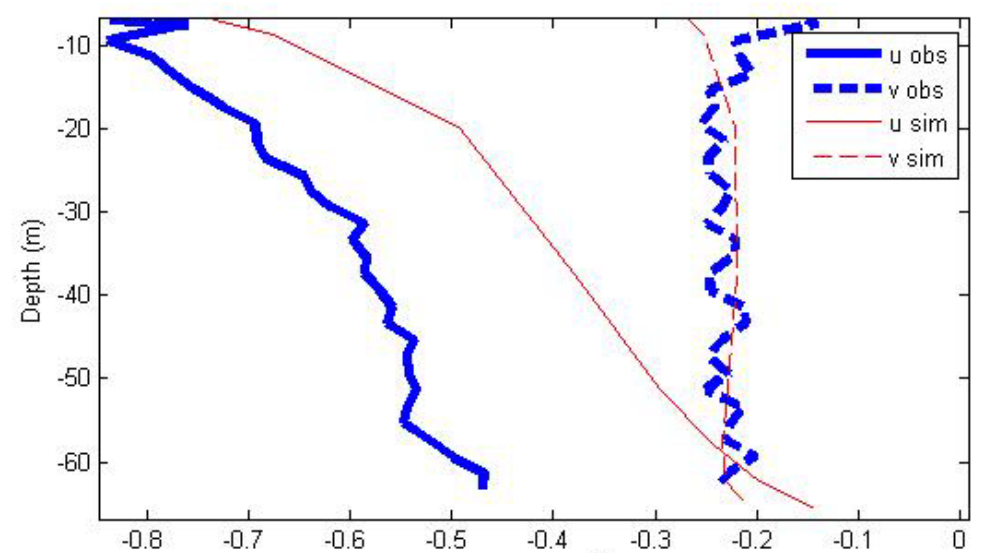

Figure 4. Profil vertical de courant à l'apex de la tempête du 18 février 2007, mesuré par la station BESSète (traits gras), simulé par le modèle de circulation océanique côtière Symphonie forcé en surface par une tension de cisaillement égale à 2,8 $\mathrm{Nm}^{-2}$ (traits fins).

\subsection{Calculs des tensions de cisaillements exercées sur le fond}

Un des intérêts de mesurer au même endroit les caractéristiques de houle et de courant consiste à pouvoir évaluer les contraintes exercées sur le fond, d'une part par les houles, d'autre part par les courants, et enfin par les effets conjugués de la houle et des courants. Même si elles peuvent être sujet à caution, les formulations de SOULSBY (1997) sont utilisées pour donner une première idée des contraintes exercées sur le fond.

La vitesse orbitale sur le fond est calculée à partir des hauteurs significatives et des périodes de pic enregistrées. Sous $70 \mathrm{~m}$ de fond, elles sont quasisystématiquement nulles, excepté pendant l'épisode de tempête où elles peuvent atteindre $0,25 \mathrm{~ms}^{-1}$ (figure 5a). Les houles interagissent alors avec le fond. Elles sont susceptibles de se transformer par réfraction et dissipation mais elles sont aussi susceptibles d'exercer une contrainte sur ce fond. Des mesures granulométriques réalisées sur des échantillons de sédiments prélevés à la benne donnent une médiane granulométrique de $10 \mu \mathrm{m}$ avec une forte prédominance de vases mais aussi la présence d’une fraction sableuse. Les tensions de cisaillement induites par la houle sont faibles mais non négligeables pendant la tempête où elles peuvent atteindre $0,16 \mathrm{Nm}^{-2}$ (figure $5 b$ ). 
Les vitesses de courants sont mesurées par l'ADCP sur toute la colonne d'eau par cellules verticales de $2 \mathrm{~m}$ d'épaisseur. La mesure ne se fait qu'à partir de la deuxième cellule au-dessus de l'appareil. En comptant l'épaisseur même du TRBM, la première mesure de courant se fait à 4,2 m au-dessus du fond (figure 5c). Malgré les forts vents de Nord (voir figure 3), l’intensité ne dépasse pas 0,2 $\mathrm{ms}^{-1}$, excepté pendant la tempête où l'intensité dépasse $0,5 \mathrm{~ms}^{-1}$. Les tensions de cisaillement induites par ces courants restent donc faibles excepté pendant la tempête où, suivant les formules de SOULSBY (1997), elles peuvent atteindre $0,25 \mathrm{Nm}^{-2}$ (figure 5d).

Pendant la tempête, le fond subit donc à la fois des contraintes unidirectionnelles (vers l'Est-Sud-Est) induites par les forts courants et des contraintes oscillantes (vers le Nord-Est) induites par les fortes houles. La résultante moyenne (sur une période de houle) calculée suivant SOULSBY (1997) est dominée par les effets du courant (figure 5e). La résultante maximale est par contre amplifiée et peut atteindre $0,3 \mathrm{Nm}^{-2}$ (figure $5 \mathrm{f}$ ). De telles valeurs sont bien sûr susceptibles de mobiliser les sédiments fins présents dans la zone et de les déplacer.

Pour aller plus loin que ce constat, il faudrait adopter une stratégie de modélisation numérique telle que celle de ULSES et al. (2008). S’appuyant sur les mêmes formulations de SOULSBY (1997) que celles utilisées ici, Ulses et al. (2008) calculent les contraintes exercées sur le fond sur l'ensemble du golfe du Lion par les effets conjugués des houles (simulées par le modèle WaveWatch III (TOLMAN, 1997)) et des courants (simulés par Symphonie). Ils en déduisent des transports et des taux d'érosion et de dépôt sur la période novembre 2003-avril 2004. L'épisode de tempête de février 2004 est relativement similaire à celui qui nous observons ici en février 2007. La zone en deçà de $70 \mathrm{~m}$ de fond apparaît pour eux globalement comme une zone d'érosion (1 cm pour l'épisode) affectée par les remises en suspension dues à la houle. Au-delà de $70 \mathrm{~m}$ de fond, l'érosion est moins nette et serait plutôt due à de forts courants qui ne dépassent toutefois pas $0,4 \mathrm{~ms}^{-1}$. Pour valider leurs résultats, ULSES et al. (2008) ne disposaient toutefois que de mesures de courants au-delà du plateau, dans les têtes de canyons sous 300 m (PALANQUES et al., 2006) ou très proche du littoral sous $30 \mathrm{~m}$ de fond à l'embouchure de la Têt (GUILLEN et al., 2006)). Pendant la tempête de février 2004, les courants simulés sur ce point sont également largement sousestimés. 

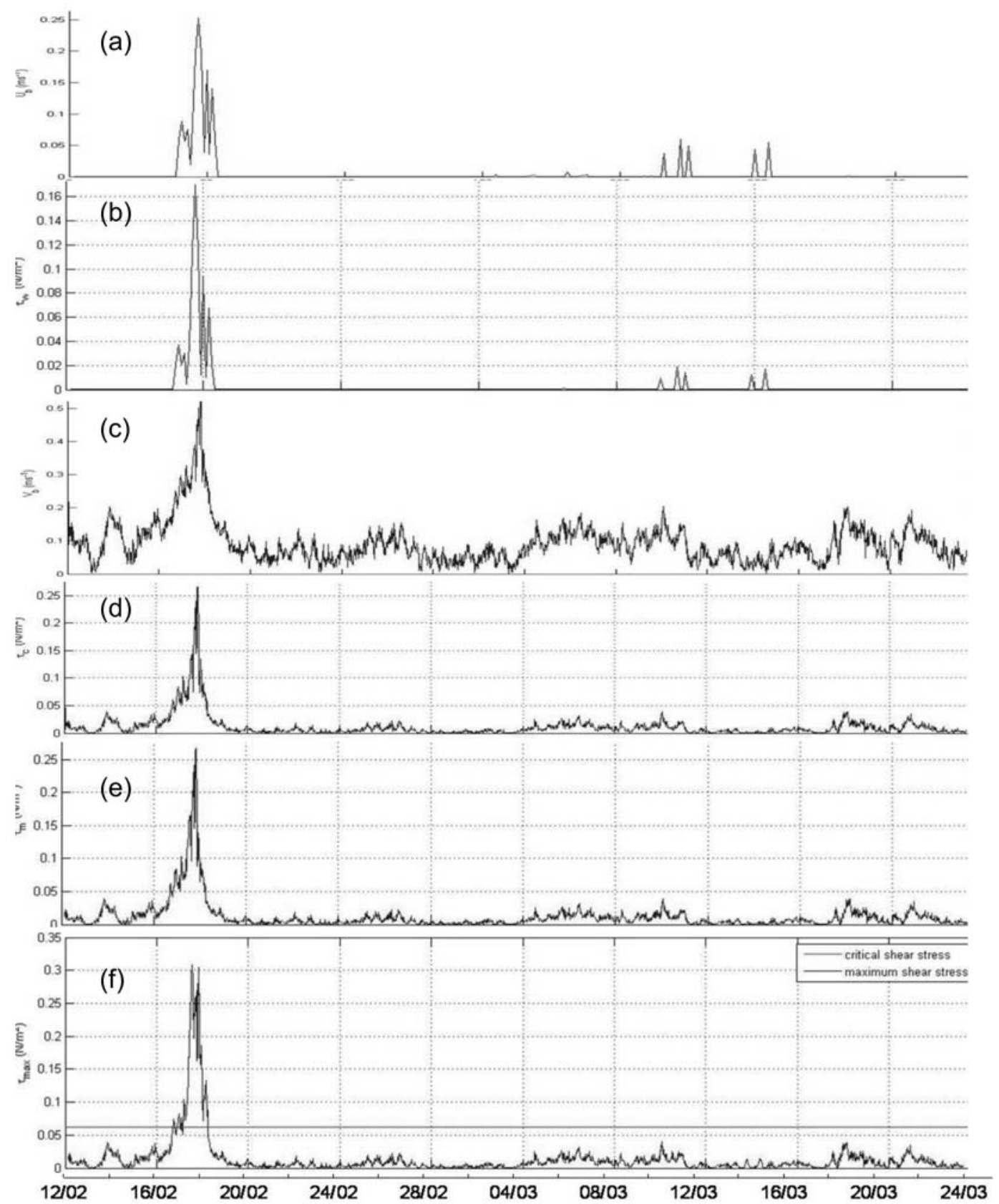

Figure 5. Série temporelle du 12 février au 25 mars 2007.

(a) Vitesse orbitale de la houle calculée près du fond (en ms${ }^{-1}$ ),

(b) tension de cisaillement exercée par la houle sur le fond (en $\left.\mathrm{Nm}^{-2}\right)$,

(c) vitesse mesurée à 4,2 $\mathrm{m}$ au-dessus du fond (en $\mathrm{ms}^{-1}$ ),

(d) tension de cisaillement exercée par les courants sur le fond (en $\mathrm{Nm}^{-2}$ ),

(e) et ( $f$ ) Tensions de cisaillement moyennes (e) et maximales ( $f$ ) exercées par les effets conjugués de la houle et des courants sur le fond (en $\mathrm{Nm}^{-2}$ ). La barre verticale représente une estimation de la tension critique de cisaillement. 


\section{Discussion sur les transferts de quantité de mouvements}

Les observations issues de la station BESSète nous amènent à réfléchir aux processus qui transfèrent d'aussi importantes quantités de mouvement de l'atmosphère vers l’océan pendant les épisodes de tempêtes (voir §2.2). Ces fortes quantités de mouvement sont par ailleurs susceptibles d'être transférées aux sédiments, pouvant ainsi impliquer de fortes mobilisations et modifications des fonds (voir §2.3).

En zone littorale (zone de surf), on se focalise en général sur les transferts de la houle vers les sédiments (e.g. CERTAIN et al., 2005). En zone côtière (sur le plateau continental), les courants sont le principal centre d'intérêt. Ces courants sont directement induits par les vents. Dans le même temps, les vents génèrent des vagues qui augmentent la rugosité de la surface et rendent ainsi plus efficace le transfert de quantité de mouvement des vents vers les courants (e.g. TAYLOR \& YELLAND, 2001). Dans le cas de la station BESSète, on a montré (§2.3 et figure 4) que pour atteindre par ce seul effet les intensités de courants observées, il faudrait atteindre des tensions de surface au moins égales à $2,8 \mathrm{~N} \mathrm{~m}^{-2}$, ce qui est complètement irréaliste.

Les courants sont également fortement induits par les gradients de pression, euxmêmes induits par les gradients d'élévation de la surface et les gradients de masse volumique, elle-même dépendante de la salinité et de la température. Les gradients de pression sont donc également dépendants des interactions avec l'atmosphère (pression atmosphérique, flux de chaleur, précipitations, fleuves...). Ces courants sont soumis à la force de Coriolis et interagissent non-linéairement par advection. La grande majorité de leur quantité de mouvement est dissipée par friction turbulente interne, mais une fraction d'autant plus grande que l'on se trouve proche du littoral va être transmise au fond. Les observations de la station BESSète montrent que cette quantité de mouvement peut-être non négligeable, $\mathrm{y}$ compris sous $70 \mathrm{~m}$ de fond, et à condition bien sûr d'être en épisode de tempête.

Dans ces conditions, les courants observés sont très forts et on peut alors se demander quels sont les processus qui induisent de si forts courants pendant ces épisodes de tempêtes. Cela peut également se traduire par la question suivante : comment injecter suffisamment de quantité de mouvement dans un modèle numérique de circulation océanique côtière pour que les courants simulés soient « réalistes » pendant les épisodes de tempête ?

Certaines pistes peuvent être étudiées :

- On a vu que l'augmentation de la rugosité de la surface par la présence de vagues peut augmenter le transfert de quantité de mouvement des vents vers les courants mais pas dans des proportions suffisantes. 
- La surélévation de la surface libre à la côte pourrait générer des courants géostrophiques. Un rapide calcul $\mathrm{U}=\mathrm{g} / \mathrm{f} * \Delta \eta / \Delta \mathrm{L}$ avec g l'accélération de la pesanteur $\left(\mathrm{g}=10 \mathrm{~ms}^{-2}\right)$, $\mathrm{f}$ paramètre de Coriolis $\left(\mathrm{f}=10^{-4} \mathrm{~s}^{-1}\right), \Delta \eta$ la dénivellation de la surface $(\Delta \eta=0.5 \mathrm{~m})$ sur une distance $\Delta \mathrm{L}(=50 \mathrm{~km})$, donne seulement une vitesse $\mathrm{U}$ égale à $0.1 \mathrm{~ms}^{-1}$.

- La circulation océanique générale pourrait également être suspectée d'injecter de la quantité de mouvement dans la zone côtière. Le Courant Nord Méditerranéen (CNM), bien que d'un débit de l'ordre de $1 \mathrm{~Sv}$, passe toutefois bien trop au Sud de la zone d'étude. Des intrusions importantes du CNM peuvent se produire beaucoup plus à l'Est du plateau (GATTI et al., 2006). D'autres intrusions du CNM ont été observées au centre (PETRENKO et al., 2005 ; LEREDDE et al., 2007) mais n’atteignent en aucun cas des intensités de courants si importantes.

Enfin les simulations numériques réalisées dans le cadre de la plateforme préopérationnelle MFSTEP (http://www.bo.ingv.it/mfstep/) ne produisent en aucun cas de si forts courants dans la zone. En particulier, la simulation, réalisée dans le cadre de ce programme par ESTOURNEL (2008, communication personnelle) pendant notre période d'observations, produit des courants, certes globalement dirigés dans la bonne direction (Est-Sud-Est) mais avec une intensité de l'ordre de $0.3 \mathrm{~ms}^{-1}$ au lieu des $0.8 \mathrm{~ms}^{-1}$ observés le 18 février 2007 à l'apex de la tempête. Les simulations réalisées par ULSES et al. (2008) pour la tempête de février 2005 confirment également cette sous-estimation des courants sur le plateau continental lors des épisodes de tempête.

Une hypothèse proposée serait alors qu'il pourrait y avoir transfert de quantité de mouvement de l'échelle de la houle vers l'échelle des courants. Très schématiquement, dès que la houle interagit avec le fond, cad dès que les houles ont des longueurs d'onde (L) supérieures à 2 fois la profondeur (H) (ici L>150 m et $\mathrm{H}=70 \mathrm{~m}$ ), la houle se transforme (réfraction, dissipation) et transmet de la quantité de mouvement à l'échelle des courants moyens. Ceci a déjà été observé pour des tempêtes sous $30 \mathrm{~m}$ de fond dans la baie de Banyuls et sur le littoral du Roussillon (DENAMIEL, 2006). C'est la première fois que l'on mettrait en évidence ce phénomène sur le plateau continental et cela nous amènerait à revoir complètement les schémas de circulation à l'échelle du plateau pendant les épisodes de tempêtes où pour le moment les vents sont considérés comme les principaux forçages des modèles de circulation océanique côtière tels que Symphonie.

Ces transferts sont bien connus et modélisés dans la zone littorale où par exemple le déferlement de la houle induit des courants entre autres de dérive littorale ou d'undertow. Certains modèles numériques comme Shorecirc (SVENDSEN et al., 
2000) prennent très bien en compte ces transferts modélisés par la notion de gradients de tensions de radiation (LONGUET-HIGGINS \& STEWART, 1962). A l'échelle côtière, sur le plateau continental, jusqu'à il y a très peu de temps, les modèles numériques de circulation océanique ignoraient systématiquement ces transferts. Cela peut être dû au manque d'observations aussi flagrantes que celles qui sont présentées ici. Cela est aussi dû aux efforts de développement pour rendre les modèles tridimensionnels (e.g. BLUMBERG \& MELLOR, 1987). Ce caractère 3D n'autorisait plus la prise en compte de pratiques aussi simples que celles des forçages par les gradients tensions de radiation. En effet, depuis LONGUET-HIGGINS \& STEWART (1962) ou PHILLIPS (1977), cette pratique n’était possible que dans les modèles 2D intégrés sur la verticale. MELLOR (2003) a relancé le sujet en écrivant un set d’équations apparentées aux équations primitives dans lesquels apparaissent des termes de forçages supplémentaires qui pour une part sont apparentés à des termes de gradients de tensions de radiation 3D. DENAMIEL (2006) a implémenté numériquement ces équations sur la base du modèle Symphonie. Les premiers résultats montrent qu'il est ainsi possible d'injecter une quantité de mouvement supplémentaire et donc de se rapprocher des valeurs de forts courants observés (DENAMIEL et al., 2005). ARDHUIN et al. (2008a) montrent toutefois que les équations de MELLOR (2003) ne sont pas cohérentes avec la présence de fonds variables. ARDHUIN et al. (2008b) proposent une alternative sous la forme des équations glm2z-RANS qui s’apparentent également aux équations primitives avec des termes de forçages supplémentaires notamment la force de vortex (LEIBOVICH, 1980). Ces équations peuvent donc également être implémentées dans un modèle numérique de circulation océanique côtière aux équations primitives (RASCLE, 2007).

Ces travaux théoriques et numériques devraient à terme permettre de modéliser les courants de manière réaliste en zone côtière pendant les épisodes de tempête et ainsi mieux représenter les transports sédimentaires induits. Le plateau du golfe du Lion, zone microtidale (les courants de marée ne viennent pas interférer avec les courants induits par les houles), large et ouverte, soumise régulièrement à des tempêtes, est particulièrement adapté à l'application de ces études.

\section{Références bibliographiques}

ANDRE G., GARREAU P., GARNIER V., FRAUNIE P. (2005). Modelled variability of the sea surface circulation in the North-western Mediterranean Sea and in the Gulf of Lions. Ocean Dynamics 55: 294-308. 
ARDHUIN F., JENKINS A.D., BELIBASSAKIS K. (2008a). Commentary on 'the three-dimensional current and surface wave equations' by George Mellor. J. Phys. Oceanogr, accepted for publication.

ARDHUIN F., RASCLE N., BELIBASSAKIS K.A. (2008b). Explicit waveaveraged primitive equations using a generalized Lagrangian mean. Ocean Modelling 20:35-60.

BLUMBERG, A. F., MELLOR G. L. (1987). A description of a threedimensional coastal ocean circulation model. Three-Dimensional Coastal ocean Models, edited by N. Heaps, 208 pp., American Geophysical Union.

CERTAIN R., MEULÉ S., REY V., PINAZO C. (2005). Wave transformation on a microtidal barred beach (Sète, France). Journal of Marine Systems 38 : 19- 34. DENAMIEL, C., BOUCHETTE, F., LEREDDE, Y., MARSALEIX, P. (2005). Three-dimensionnal coupled modeling of wave- and wind/buoyancy-driven currents: An application in the Gulf of Aigues-Mortes (NW Mediterranean sea, France). European Geosciences Union, General Assembly. Vienna, Austria, 24 29 April 2005. Geophysical Research Abstracts, 7, 03268.

DENAMIEL C. (2006). Modélisation hydrodynamique 3D en zone pré-littorale : Caractérisation des effets des houles de tempête sur la circulation océanique. Thèse de l’Université Montpellier 2. 424 pp.

ESTOURNEL C., DURRIEU DE MADRON X., MARSALEIX P., AUCLAIR F., JULLIAND C., VEHIL R. (2003). Observation and modelisation of the winter coastal oceanic circulation in the gulf of lions under wind conditions influenced by the continental orography (fetch experiment). Journal of Geophysical Research, 108(C3) :8059.

GATTI J., PETRENKO A., LEREDDE Y., DEVENON J.L. (2006). Modelling the intrusions of the Northern Current on the eastern part of the Gulf of Lion's continental shelf. European Geosciences Union, General Assembly 2006, Vienne, Autriche, 02 - 07 Avril 2006. Geophysical Research Abstracts, 8, 00684.

GREMARE A., AMOUROUX J. M., CAUWET G., CHARLES F., COURTIES C., DE BOVEE F., DINET A., DEVENON J. L., DURRIEU DE MADRON X., FERRE B., FRAUNIE P., JOUX F., LANTOINE F., LEBARON P., NAUDIN J. J., PALANQUES A., PUJO-PAY M., ZUDAIRE L. (2003). The effects of strong winter storm on physical and biological variables at a shelf site in the mediterranean. Oceanologica acta, 26 :407-419.

GUILlEN J., BOURRIN F., PALANQUES A., DURIEU DE MADRON X., PUIG P., BUSCAIL R. (2006). Sediment dynamics during "wet" and "dry" storm events on the tet inner shelf (sw gulf of lions). Marine Geology, 234, 1-4 :129-142. 
LEIBOVICH S. (1980). On wave-current interaction theory of Langmuir circulations. J. Fluid Mech. 99, 715-724.

LEREDDE Y., DENAMIEL C., BRAMBILLA E., LAUER-LEREDDE C., BOUCHETTE F., MARSALEIX P. (2007). Hydrodynamics in the Gulf of AiguesMortes, NW Mediterranean Sea: in situ and modelling data. Continental Shelf Researh, 27 2389-2406.

LONGUET-HIGGINS M.S., STEWART R.W. (1962). Radiation stresses; a physical discussion, with applications. Deep-Sea Research 11, 529-562.

MELLOR G., 2003. The three dimensional current and surface wave equations. Journal of Physical Oceanography, 33:1978-1989.

MILLOT, C. (1990). The gulf of Lion's hydrodynamics. Continental Shelf Research, 10(9-11) :885-894.

PALANQUES A., DURRIEU DE MADRON X., PUIG P., FABRES J., GUILLEN J., CALAFAT A., CANALS M., BONNIN J. (2006). Suspended sediment fluxes and transport processes in the Gulf of Lions submarine canyons. The role of storms and dense water cascading. Marine Geology 234, 41-63.

PETRENKO A., LEREDDE Y., MARSALEIX P. (2005). Circulation in a stratified and wind-forced gulf of Lions, NW Mediterranean sea : in-situ and modeling data. Continental Shelf Research, 319, 25 :7-27.

PHILIPS O.M. (1977). The Dynamics of the Upper Ocean. Cambridge University Press, 2 d edition. 328 pp.

RASCLE N. (2007). Impact des vagues sur la circulation océanique. Thèse de l’Université Bretagne Occidentale, 225 pp.

SOULSBY R. (1997). Dynamics of marine sands. Thomas Telford. 245 pp.

SVENDSEN I.A., HAAS K., ZHAO Q. (2000). Quasi-3D nearshore circulation model, SHORECIRC, Version 1.3.6, Report, Center for Applied Coastal Research. University of Delaware, Newark.

TAYLOR P.K., YELLAND M.J. (2001). The dependance of the sea surface roughness on the height and steepness of the waves. Journal of Physical Oceanography, $31: 572-590$.

TOLMAN H.L., 1997. User manual and system documentation of Wavewatch III version 1.15.

ULSES C., ESTOURNEL C., DURRIEU DE MADRON X., PALANQUES A. (2008). Suspended sediment transport in the Gulf of Lions (NW Mediterranean): Impact of extreme storms and floods. Continental Shelf Research, sous presse. 